\title{
Temporal adjustment of the juxtaglomerular apparatus during sustained inhibition of proximal reabsorption
}

\author{
Scott C. Thomson, ${ }^{1}$ Sebastian Bachmann, ${ }^{2}$ Magdalena Bostanjoglo, ${ }^{2}$ \\ Carolyn A. Ecelbarger, ${ }^{3}$ Orjan W. Peterson, ${ }^{1}$ Doron Schwartz, ${ }^{1}$ Dingjiu Bao, ${ }^{1}$ \\ and Roland C. Blantz ${ }^{1}$ \\ ${ }^{1}$ Department of Medicine, Division of Nephrology/Hypertension, University of California School of Medicine; \\ Veterans Affairs Medical Center; and Veterans Medical Research Foundation; San Diego, California 92161, USA \\ ${ }^{2}$ Institut für Anatomie, Charité, Humboldt-Universität, D-14050 Berlin, Germany \\ ${ }^{3}$ Laboratory of Kidney and Electrolyte Metabolism, National Heart, Lung, and Blood Institute, National Institutes of Health, \\ Bethesda, Maryland 20892, USA
}

Address correspondence to: Scott C. Thomson, Division of Nephrology/Hypertension, University of California-San Diego and San Diego Veterans Affairs Medical Center, 3350 La Jolla Village Drive, San Diego, California 92161-9111H, USA. Phone: (619) 552-7528; Fax: (619) 552-7549; E-mail: sthomson@ucsd.edu.

This work was presented in abstract form before the American Society of Nephrology; in San Antonio, Texas, USA, in November 1997.

Received for publication September 8, 1998, and accepted in revised form August 31, 1999.

\begin{abstract}
Tubuloglomerular feedback (TGF) stabilizes nephron function by causing changes in single-nephron GFR (SNGFR) to compensate for changes in late proximal flow (VLP). TGF responds within seconds and reacts over a narrow range of VLP that surrounds normal VLP. To accommodate sustained increases in VLP, TGF must reset around the new flow. We studied TGF resetting by inhibiting proximal reabsorption with benzolamide (BNZ; administered repeatedly over a 24-hour period) in Wistar-Froemter rats. BNZ acutely activates TGF, thereby reducing SNGFR. Micropuncture was performed 6-10 hours after the fourth BNZ dose, when diuresis had subsided. BNZ caused glomerular hyperfiltration, which was prevented with inhibitors of macula densa nitric oxide synthase (NOS). Because of hyperfiltration, BNZ increased VLP and distal flow, but did not affect the basal TGF stimulus (early distal salt concentration). BNZ slightly blunted normalized maximum TGF response and the basal state of TGF activation. BNZ sensitized SNGFR to reduction by $S$-methyl-thiocitrulline (SMTC) and caused the maximum TGF response to be strengthened by SMTC. Sensitization to type I NOS (NOS-I) blockers correlated with increased macula densa NOS-I immunoreactivity. Tubular transport measurements confirmed that BNZ affected TGF within the juxtaglomerular apparatus. During reduced proximal reabsorption, TGF resets to accommodate increased flow and SNGFR through a mechanism involving macula densa NOS.
\end{abstract}

J. Clin. Invest. 104:1149-1158 (1999).

\section{Introduction}

In the mammalian kidney, there is close coordination between the processes of glomerular filtration and tubular reabsorption. This arrangement prevents small changes in GFR or inefficiencies in reabsorption from resulting in large changes in solute and water excretion. Along with glomerular-tubular balance (GTB), coordination between the glomerulus and tubule is mediated by a system of tubuloglomerular feedback (TGF), which operates within the juxtaglomerular apparatus (JGA) of each nephron. The JGA comprises the macula densa (a specialized segment of tubular epithelium at the end of the loop of Henle), the vascular pole of the glomerulus, and the extraglomerular mesangium (interposed between the macula densa and the glomerular vascular pole). The TGF process consists of a signaling step and an effector step. In the signaling step, the macula densa senses changes in salt content of tubular fluid. In the effector step, vasoconstriction or vasodilation of the glomerular arterioles causes changes in the singlenephron GFR (SNGFR), which compensates for changes in the signal detected by the macula densa. The TGF system operates on a time scale of several seconds. The degree to which a TGF response succeeds in offsetting a particular disturbance in tubular flow is determined by the "gain" or "efficiency" of the TGF system.

TGF causes an inverse dependence of SNGFR on the macula densa signal, which is markedly nonlinear and characterized by a narrow, steep portion that tends to be centered about the ambient signal in any given nephron (1). Because the TGF gain is proportional to the steepness of this slope, the ability of TGF to stabilize nephron function is quite sensitive to changes in this alignment between TGF and the ambient signal. Whereas the TGF relationship itself is nonlinear, the salt content of tubular fluid reaching the macula densa varies in direct proportion to the rate of flow in the late proximal tubule. Therefore, when TGF is studied in the laboratory, late proximal flow (VLP) is usually employed as a surrogate for the salt content at the macula densa. The tendency for TGF to be aligned with the natural tubular flow suggests that some mechanism might exist to coordinate the TGF process with tubular flow at the level of the individual nephron. In fact, when VLP is tonically increased for 30-60 minutes in a single nephron, the peak gain shifts rightward and realigns with the newly 
imposed VLP (2). Therefore, the TGF system can adapt to maintain its own efficiency, and the chain of events responsible for this adaptation can be demonstrated within the individual nephron. The phenomenon whereby TGF adapts to accommodate long-term changes in VLP is referred to as TGF resetting.

Beyond the fact that it can be mediated entirely within a single nephron, little is known about the mechanism of TGF resetting. Furthermore, it is possible that the events that initiate TGF resetting within the first hour are different from those that cause it to remain reset hours or days later. For instance, suppose that a sustained increase in renal perfusion pressure or a decrease in proximal tubular reabsorption were suddenly imposed, thereby increasing VLP and activating TGF. Within 1 hour, TGF would reset. Resetting on this time scale most likely results from chemical-hormonal changes in the JGA that restore TGF efficiency without requiring major alterations in cell structure or transport capacity. However, if increased delivery into the loop of Henle were to persist for several hours, this might allow time to increase transport capacity in the loop of Henle, which would allow conditions at the macula densa to return to normal while maintaining TGF efficiency at the new VLP. Also, whereas the immediate dependence of SNGFR on VLP may appear to be the same whether the nephron has been exposed to a resetting stimulus for 1 hour or for many hours, it is possible that the humoral-cellular interactions within the JGA that maintain this relationship continue to evolve over time, becoming more permanent. A more permanent resetting could allow SNGFR to exceed its original value and to remain elevated for some time after the stimulus to reset was removed.

In this study, TGF resetting was examined in a series of experiments in which proximal reabsorption was suppressed by repeatedly injecting rats with the carbonic anhydrase inhibitor benzolamide (BNZ) over a period of 24 hours. Resetting of TGF over this 24-hour period led to persistent glomerular hyperfiltration, as confirmed in whole-kidney clearance studies and by micropuncture performed 6-10 hours after the final dose of BNZ was administered. Further physiologic and immunohistochemical studies were performed to determine whether the process of TGF resetting during 24 hours of BNZ involves upregulation of type I nitric oxide synthase (NOS-I) in the macula densa. Finally, micropuncture and immunoblotting experiments were conducted to ascertain whether the apparent resetting of TGF during 24 hours of BNZ could be accounted for by a primary increase in loop of Henle transport, i.e., a reduced macula densa signal at any given VLP. This analysis involved testing for changes in the $\mathrm{Na} / \mathrm{K} / 2 \mathrm{Cl}$ transporter, BSC-1, and the proximal tubule/descending limb water channel, aquaporin-1 (AQP1). Data generated in the course of these experiments demonstrate that, when repeatedly exposed to BNZ over a period of 24 hours, the kidney adapts to accommodate increased tubular flow through a rightward and upward shift in the TGF relationship. This resetting of TGF may depend on increased formation of NO in the macula densa, and does not require a primary increase in loop of Henle transport. The temporal adaptation of TGF is potentially an important component of the renal adaptation to externally imposed changes in GFR, tubular reabsorption, and major alterations in sodium chloride $(\mathrm{NaCl})$ intake and volume status.

\section{Methods}

All animal experimentation was conducted in accordance with the National Institutes of Health (NIH) Guide for the Care and Use of Laboratory Animals. Studies were performed in male Wistar-Froemter rats that were bred and housed in the Veterinary Medical Unit at the Veterans Affairs Medical Center (San Diego, California, USA) and weighed between 250 and $330 \mathrm{~g}$ at the time of the study. Animals received free access to tap water and standard rat chow. Studies were performed in rats in which sustained activation of TGF was achieved by the proximal tubular diuretic, BNZ ( $5 \mathrm{mg} / \mathrm{kg}$ intraperitoneally every 6 hours for 4 doses). BNZ acutely reduces proximal reabsorption by approximately $50 \%$, thereby increasing fluid entry to the loop of Henle, with subsequent activation of TGF. Administered acutely, BNZ causes a TGF-mediated reduction in GFR (3). (In pilot studies performed in awake rats, single intraperitoneal doses of BNZ exerted a diuretic effect that lasted less than 6 hours.). In some animals, each dose of $\mathrm{BNZ}$ was administered along with the specific inhibitor of NOS-I, 7-nitroindazole (7-NI; $40 \mathrm{mg} / \mathrm{kg}$ subcutaneously in DMSO), to inhibit macula densa NOS. Control animals received saline placebo or DMSO in lieu of $\mathrm{BNZ}$ or 7-NI, as appropriate.

Surgical preparation for micropuncture. Micropuncture was performed under Inactin $(100 \mathrm{mg} / \mathrm{kg}$ intraperitoneally; Andrew Lockwood Associates, Ann Arbor, Michigan, USA) anesthesia after tracheostomy (PE 240) and catheterization (PE 50) of the right jugular vein, left femoral artery, urinary bladder, and left ureter, and after surgical preparation of the left kidney for micropuncture, according to protocols described previously in publications from this laboratory (4). Body temperature was regulated by a servo-controlled heating table. All micropuncture was conducted under euvolemic conditions, with animals receiving donor rat plasma $(11 \mathrm{~mL} / \mathrm{kg}$ body weight over 60 minutes, followed by continuous infusion at $2 \mathrm{~mL} / \mathrm{kg}$ body weight per hour) as replacement for surgical losses. An additional infusion of Ringer's saline solution containing $80 \mu \mathrm{Ci} / \mathrm{mL}\left[{ }^{3} \mathrm{H}\right]$ inulin as a marker of glomerular filtration was infused continuously at $1.5 \mathrm{~mL} / \mathrm{h}$. After completion of the preparatory surgery, animals were allowed 60 minutes to equilibrate before beginning micropuncture. Arterial blood pressure was monitored from the femoral artery catheter using a P23Db Gould Statham pressure transducer and Statham chart recorder.

Micropuncture assessment of SNGFR and tubular reabsorption after BNZ. For purposes of this study, "natural" values for SNGFR and VLP are defined as those values that are the result of the normal operation of TGF. When SNGFR and VLP are measured by collecting fluid from the late proximal tubule, TGF is interrupted, and the values obtained for SNGFR and VLP will exceed the natural values. Natural SNGFR (SNGFRd) was determined by inulin clearance in timed samples of fluid from early distal nephrons by micropuncture. The corresponding 
natural VLP was computed from SNGFRd, and the concentration of inulin in fluid sampled from the late proximal tubule of that nephron relative to the concentration of inulin in systemic plasma. Early distal flow rate was determined volumetrically. Proximal reabsorption was computed from SNGFRd and VLP. Loop of Henle reabsorption was computed from VLP and early distal flow rate. The ionic content of fluid in the early distal nephron (a surrogate for the salt content of fluid reaching the macula densa) was measured using a conductivity microelectrode placed in the early distal nephron as described previously (5). The tonic influence of TGF over SNGFR was determined from the paired proximal-distal difference (PDD) in SNGFR. PDD is the difference between inulin clearance in fluid collected from the proximal tubule (TGF being inactivated by the act of collecting upstream from the macula densa) and SNGFRd (TGF operating normally). To test for changes in the capacity for loop of Henle reabsorption, microperfusion studies were performed using a Hample microperfusion apparatus to deliver artificial tubular fluid (ATF) into the loop of Henle from the late proximal nephron. Standard and high-bicarbonate ATFs were employed, with standard ATF containing (in $\mathrm{mM}$ ): $130 \mathrm{NaCl}, 10$ $\mathrm{NaHCO}_{3}, 4 \mathrm{KCl}, 2 \mathrm{CaCl}_{2}, 45 \mathrm{mg} /$ percent urea, $0.1 \%$ FD\&C green ( $\mathrm{pH} 7.4)$. Standard ATF was altered to make high-bicarbonate ATF by increasing $\mathrm{NaHCO}_{3}$ to $25 \mathrm{mM}$ and reducing $\mathrm{NaCl}$ to $115 \mathrm{mM}$. The effect of perfusion rate on loop of Henle fluid flux was determined by micropuncture collection from the early distal tubule. $\left[{ }^{3} \mathrm{H}\right]$ inulin was added tothe ATF, and the microperfusion rate was calculated from the inulin content of the fluid collected. The effect of perfusion rate on loop of Henle solute flux was determined by measuring electrical conductivity in the early distal nephron. In these experiments, the microperfusion pump was calibrated in vitro.

Role of macula densa NOS in the adaptation to BNZ. After glomerular hyperfiltration was found to occur during hours 6-10 after a fourth dose of BNZ, 2 additional sets of physiologic experiments were performed to ascertain whether macula densa NOS is critical to this phenomenon. In the first set of experiments, BNZ and control animals were treated with the specific inhibitor of NOS-I, 7$\mathrm{NI}(40 \mathrm{mg} / \mathrm{kg}$ subcutaneously in $200 \mu \mathrm{L}$ DMSO every 6 hours for 4 doses), or with DMSO alone. To control for the effect of diuresis per se on the interaction between BNZ and 7-NI, animals were weighed every 6 hours, and any decrement in weight from the previous weighing was replaced with Ringer's saline solution administered subcutaneously. Eight hours after the final dose of BNZ (with or without 7-NI) animals were subjected to surgery using the same method as for micropuncture, except that the abdomen was not opened and the replacement fluid consisted of Ringer's saline at $2 \mathrm{~cm}^{3} / \mathrm{h}$. After 1 hour of postsurgical equilibration, GFR was measured by $\left[{ }^{3} \mathrm{H}\right]$ inulin clearance in 2 consecutive 30 -minute periods. Urine flow was determined by the weight of the urine collected.

In the second set of experiments, the role of macula densa NOS in the post-BNZ period was studied by micropuncture. BNZ and control animals were prepared for micropuncture according to the protocol described above. No NOS inhibitor was given before micropuncture.
Instead, macula densa NOS was inhibited at the singlenephron level by microperfusion with the water-soluble NOS-I blocker, S-methyl-thiocitrulline (SMTC; $1 \mathrm{mM}$ in standard ATF). The relative importance of underlying macula densa NOS activity as a determinant of SNGFR in $\mathrm{BNZ}$ and control rats was determined by measuring SNGFR and the range over which SNGFR could be made to change by manipulating TGF. This was accomplished by measuring $\left[{ }^{3} \mathrm{H}\right]$ inulin clearance in late proximal collections during orthograde perfusion of the loop of Henle at 8 or $38 \mathrm{~nL} / \mathrm{min}$ with standard ATF or ATF + SMTC. To strengthen the statistical analysis, each nephron was studied at both perfusion rates with both perfusates. Perfusions were in the following order: ATF at $38 \mathrm{~nL} / \mathrm{min}$, ATF at $8 \mathrm{~nL} / \mathrm{min}, \mathrm{ATF}+\mathrm{SMTC}$ at $38 \mathrm{~nL} / \mathrm{min}, \mathrm{ATF}+\mathrm{SMTC}$ at $8 \mathrm{~nL} / \mathrm{min}$. The $38 \mathrm{-nL} / \mathrm{min}$ perfusions were continued for 4 minutes before timed collections to allow time for SMTC to permeate the macula densa. The $8-\mathrm{nL} / \mathrm{min}$ perfusions were continued for 2 minutes before timed collections to allow equilibration of TGF while minimizing the chances for the amount of SMTC at the macula densa to be reduced at the lower perfusion rate. Between collections, a pipette was maintained in the late proximal tubule so that glomerular filtration could continue. Timed collections were from 2.5 to 3 minutes.

Preparation of kidneys for in vitro analyses. Rats were anesthetized with pentobarbital sodium $(40 \mathrm{mg} / \mathrm{kg}$ body weight intraperitoneally), perfused in a retrograde fashion through the abdominal aorta with $45 \mathrm{~mL}$ freshly prepared 3\% paraformaldehyde in PBS at $\mathrm{pH} 7.4$, and then rinsed with a sucrose-PBS solution adjusted to 800 $\mathrm{mOsm} / \mathrm{kg}$ ( 5 minutes, $60 \mathrm{mmHg}$ ). Kidneys were then removed and cut into $3-\mathrm{mm}$-thick slices that were immediately frozen in liquid nitrogen-cooled isopentane. Frozen specimens were sent to Berlin for analysis of NOS-I content, and to NIH for analysis of BSC-1.

Macula densa NOS-I by immunocytochemistry and in situ bybridization. Expression of mRNA for NOS-I was localized using digoxigenin-labeled riboprobes made from cDNA by incorporation of DIG-UTP. The probe for detection of NOS-I mRNA was transcribed from a NOSI subclone containing a PstI fragment (position 2351-3423) from the original NOS-I clone (6). To generate the antisense probes, plasmid was linearized by SacX and transcribed by T3 polymerase. A NOS-I sense probe for control was not included, because location of the signal and specificity of the detection have been wellestablished in previous work (7). DIG-UTP-labeled NOS-I probes were subjected to time-controlled alkaline hydrolysis according to standard methodology. Probes were checked by agarose gel electrophoresis and ethidium bromide staining. For in situ hybridization, cryostat sections were fixed in $4 \%$ paraformaldehyde, acetylated with $0.1 \mathrm{M}$ triethanolamine containing acetic anhydride, and then incubated with the prehybridization and hybridization mixture as described (8). Particular attention was given to keeping the thickness of the sections constant. Concentrations of the labeled probe were between 9 and $12 \mathrm{ng} / \mu \mathrm{L}$ of hybridization mixture, with $20 \mu \mathrm{L}$ applied per section. The sections were hybridized at $40^{\circ} \mathrm{C}$ for $18-20$ hours. Slides were washed in $2 \times$ SSC without formamide for 30 minutes, followed by several 
washes at $40^{\circ} \mathrm{C}$ in decreasing concentrations of SSC containing 50\% formamide. Subsequently, slides were rinsed in $0.1 \times$ SSC at room temperature for 20 minutes, followed by two 10 -minute rinses; they were then processed for detection using alkaline phosphatase-labeled Fab fragments and a detection solution containing nitroblue tetrazolium, X-phosphate (5-bromo-4-chloro-3-indolyl phosphate), and $N$ - $N$-dimethylformamide. The reaction was stopped by immersing slides in tris (hydroxymethyl)aminomethane hydrochloride containing EDTA, when a clear signal at the JGA was obvious and background staining had not yet developed. For NOS-I mRNA detection, a standardized time for color development was set at 72 hours (incubation temperature: $4^{\circ} \mathrm{C}$ ). Slides were rinsed in PBS and mounted under coverslips with phosphate-buffered glycerol.

For the detection of NOS-I protein by immunostaining, an antibody to NOS-I was provided by Bernd Mayer (Institut für Pharmakologie und Toxikologie, KarlFranzens-Universität Graz, Graz, Austria). Nonspecific antibody binding sites were blocked by incubating sections with a mixture of normal sheep serum, BSA, and Triton X-100. Secondary antibody coupled to horseradish peroxidase or alkaline phosphatase was applied for signal detection using standard color-generating solution and synchronized protocols. Immunostaining was controlled by replacing the specific antibody with nonimmune serum. Structural resolution was sufficient to identify each immunoreactive cell in each glomerulus. Findings were expressed as the number of positive macula densa cells per 100 glomeruli.

Contents of $\mathrm{Na} / \mathrm{K} / 2 \mathrm{Cl}$ transporter and aquaporin proteins. Frozen kidneys were thawed and homogenized using a polytron homogenizer. Protein content of the homogenate was measured by the Lowry technique (9). Samples of the whole homogenates were prepared for Western blotting as described previously (10), by solubilizing an aliquot of the homogenate at $60^{\circ} \mathrm{C}$ for 15 minutes in Laemmli sample buffer.

SDS-PAGE of solubilized whole-kidney protein was carried out on precast Mini-Gels (Bio-Rad Laboratories Inc., Hercules, California, USA) of $7.5 \%$ acrylamide, using a
Mini-PROTEAN II Electrophoresis System (Bio-Rad). The proteins were electrophoretically transferred from the gels to nitrocellulose membranes using a Bio-Rad Mini Trans-Blot Electrophoretic Transfer Cell. The nitrocellulose membranes were blocked for 30 minutes in 5\% milk and then were probed overnight at $4{ }^{\circ} \mathrm{C}$ with the affinity-purified rabbit polyclonal antibodies directed against rat BSC-1, AQP1, or AQP2 (11). Antibodies were diluted into a solution containing $150 \mathrm{mM} \mathrm{NaCl}, 50 \mathrm{mM}$ sodium phosphate, $10 \mathrm{mg} / \mathrm{dL}$ sodium azide, $50 \mathrm{mg} / \mathrm{dL}$ Tween-20, and $0.1 \mathrm{~g} / \mathrm{dL}$ BSA ( $\mathrm{pH} 7.5$ ). The secondary antibody was donkey anti-rabbit IgG conjugated to horseradish peroxidase (Pierce Chemical Co., Rockford, Illinois, USA) used at a concentration of $0.16 \mu \mathrm{g} / \mathrm{mL}$. To view sites of antibody-antigen reaction, blots were exposed to a Luminol-based enhanced chemiluminescence reagent (LumiGlo; Kirkegaard \& Perry Laboratories Inc., Gaithersburg, Maryland, USA) before exposure to xray film (catalog no. 165-1579; Eastman Kodak Scientific Imaging Systems, Bridgeport, Connecticut, USA).

Statistics. Intergroup comparison of unpaired freeflow micropuncture data was by Student's $t$ test. Intergroup differences in the outcome of microperfusion experiments to test for changes in loop of Henle reabsorption were examined by the multivariate general linear model, with microperfusion rate and experimental group treated as parametric and categorical independent variables, respectively. The effect of BNZ on the maximum range of the TGF response was performed by applying 1-way ANOVA with repeatedmeasures design to values for SNGFR obtained during loop perfusion with ATF at 8 and $38 \mathrm{~nL} / \mathrm{min}$. The effects of BNZ on the role of macula densa NOS as a determinant of SNGFR were tested by ANOVA with repeated measures design where the effect within subjects was taken to be the effect of SMTC on SNGFR. Separate statistical comparisons were performed for SNGFR at each of the 2 perfusion rates and for SNGFR at the TGF midpoint. The midpoint occurs where the TGF response is half of maximum; SNGFR at this point is obtained simply by averaging the SNGFRs for the 2 perfusion rates. The effects of BNZ

\section{Figure 1}

Micropuncture results. Tukey box plots of (a) SNGFR collected from the early distal tubule (SNGFRd), (b) VLP calculated from SNGFRd and late proximal tubular fluid/plasma inulin, (c) early distal flow, and (d) the ionic content of the early distal tubule normalized to ATF. Measurements were made 6-8 hours after a fourth dose of BNZ. Each box is centered about the mean. The ends of a box indicate first and third quartiles; the line within the box indicates the median; the error bars indicate $10-90 \%$ confidence intervals; and the circles represent individual outliers.

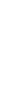

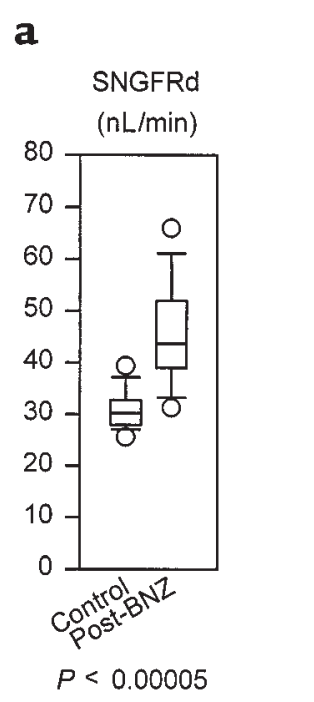

b

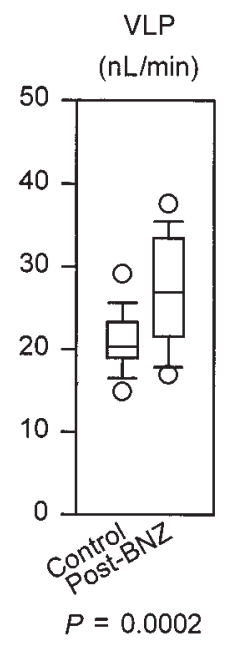

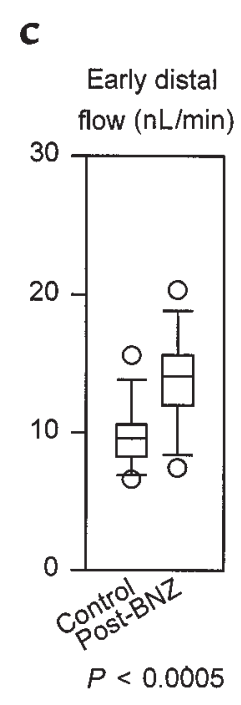

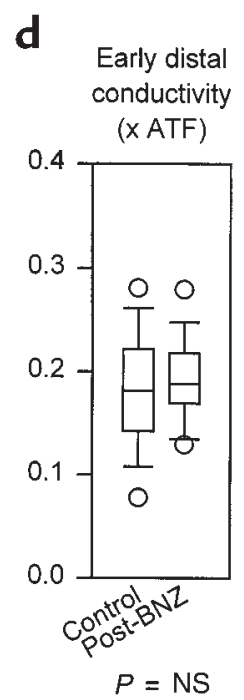




\section{Figure 2}

Proximal tubule and loop of Henle reabsorption. Tukey box plots of (a) fractional proximal reabsorption and absolute proximal reabsorption, (b) fractional loop of Henle reabsorption and absolute loop of Henle reabsorption, and (c) PDD in SNGFR measured 6-8 hours after a fourth dose of BNZ. >
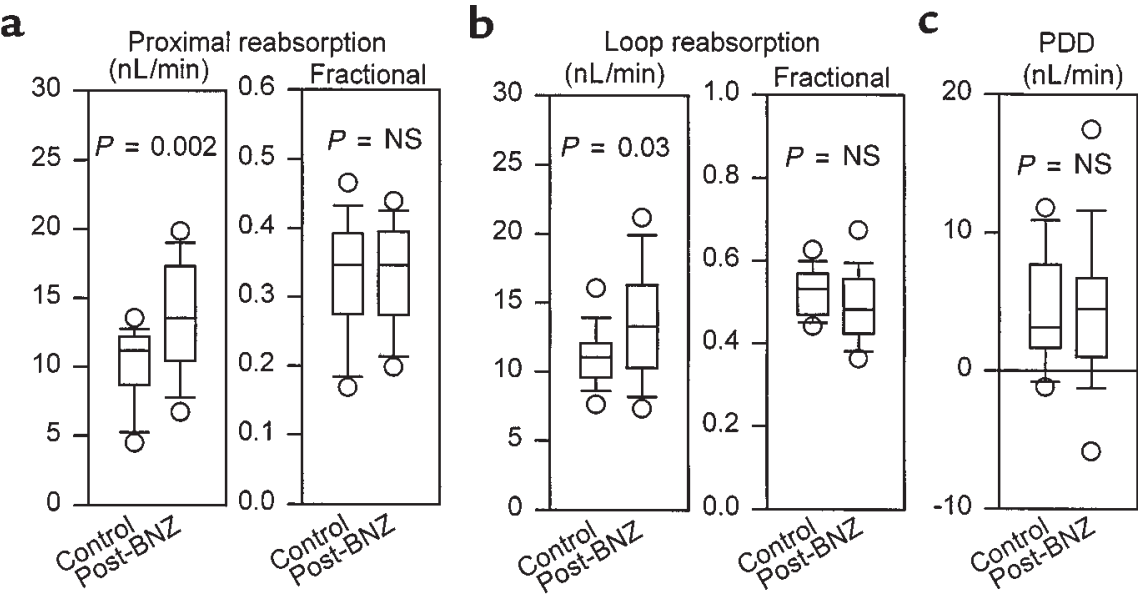

and 7-NI on whole-kidney GFR were tested by 2-way ANOVA. The effect of BNZ on results of immunohistochemistry and in situ hybridization for NOS-I was tested by Lord score. The effect of BNZ on BSC-1 was tested by unpaired Student's $t$ test.

\section{Results}

Initial data on distally collected SNGFR and tubular reabsorption were collected from 69 nephrons in 10 rats $(5$ control and 5 post-BNZ treatment). In these animals, previous treatment with BNZ did not affect hematocrit $(0.48 \pm 0.01$ vs. $0.47 \pm 0.01)$, mean arterial blood pressure ( $115 \pm 3$ vs. 109 $\pm 4 \mathrm{mmHg})$, or left-kidney urine flow $(3.5 \pm 0.2$ vs. $4.1 \pm 0.9$ $\mathrm{uL} / \mathrm{min})$. SNGFRd, VLP, and early distal flow rate were significantly greater in nephrons from post-BNZ rats than from control rats, whereas previous treatment with BNZ did not affect the ambient ionic content of the early distal nephron (Figure 1). Absolute reabsorption from the proximal tubule and loop of Henle in post-BNZ rats was increased in proportion to the delivered load, such that fractional reabsorption of water in these segments was the same in both groups (Figure 2). The tonic influence of TGF as a determinant of SNGFR was calculated from the PDD in SNGFR. In absolute terms, PDD did not differ between control animals and those previously exposed to BNZ $(P=$ 0.40; Figure 2). When expressed as a fraction of SNGFRd, there was a tendency for PDD to be less in post-BNZ rats (0.119 vs. $0.179 ; P=0.09)$. The maximum range over which TGF could cause SNGFR to change was assessed by microperfusion and equated to the increment in proximally collected SNGFR, which occurred as the loop of Henle perfusion rate was reduced from $38 \mathrm{~nL} / \mathrm{min}$ (maximum TGF) to $8 \mathrm{~nL} / \mathrm{min}$ (minimum TGF). In these same experiments, midpoint values for SNGFR were calculated by averaging the 2 measurements made in each nephron. Data were obtained in 31 nephrons from 8 rats $(4$ control and 4 post-BNZ rats) (Figure 3). Control and BNZ rats did not differ with respect to weight $(261 \pm 6$ vs. $269 \pm 7 \mathrm{~g}$ ) or blood pressure ( $123 \pm 3$ vs. $127 \pm 3 \mathrm{mmHg}$ ). Results obtained during perfusion with ATF at $8 \mathrm{~nL} / \mathrm{min}$ confirmed the results of the PDD experiments described above, in that SNGFR was significantly greater in post-BNZ animals. Previous exposure to BNZ was also associated with greater SNGFR during maximal TGF stimulation (see Figure 3 ) and at the TGF midpoint (50 \pm 4 vs. $33 \pm 3 \mathrm{~nL} / \mathrm{min} ; P=0.002)$. In absolute terms, previous treatment with BNZ did not appear to affect the maximum range of TGF (14 \pm 3 vs. 17 $\pm 4 \mathrm{~nL} / \mathrm{min} ; P=0.50)$; however, when the range of the TGF response was expressed as a fraction of SNGFR at the TGF midpoint, there was an apparent tendency for BNZ to reduce the maximum TGF response $(0.325 \pm 0.094$ vs. 0.603 $\pm 0.136 ; P=0.08$; Figure 3 ).

The role of macula densa NOS-I in the renal adaptation to $\mathrm{BNZ}$ was tested by whole-kidney $\left[{ }^{3} \mathrm{H}\right]$ inulin clearance in 24 rats weighing $268 \pm 6 \mathrm{~g}$. Respective arterial blood pressure values for control, 7-NI, BNZ, and BNZ + 7-NI were $114 \pm 3,122 \pm 9,121 \pm 8$, and $126 \pm 4 \mathrm{mmHg}$ (mean \pm SEM). The tendency for 7-NI to increase blood pressure was not significant ( $P=0.30$ by 2 -way ANOVA). Over the 24 hours of treatment before clearance measurements, body weight did not change in rats receiving placebo. Over same 24-hour period, rats receiving BNZ lost $6.8 \pm 2.9 \mathrm{~g}$ body weight, and rats receiving BNZ + 7-NI lost $11.0 \pm 2.6$ g body weight. Hematocrit was $51 \pm 0.2 \%, 49 \pm 1 \%$, and 51 $\pm 1 \%$ in control, BNZ, and BNZ +7 -NI rats $(P=\mathrm{NS})$, respectively. Urine flow rate was (in $\mu \mathrm{L} / \mathrm{min}$ ) $16 \pm 3,12 \pm$ 3 , and $11 \pm 2$ in control, BNZ, and BNZ +7 -NI rats $(P=$ NS), respectively. There was no significant effect of 7-NI on GFR in animals not receiving BNZ (2.7 \pm 0.4 vs. $2.2 \pm$ $0.1 \mathrm{~mL} / \mathrm{min} ; P=0.20)$, although the $7-\mathrm{NI}$ group had large variance. Adding 7-NI reduced GFR in BNZ rats $(1.7 \pm 0.2$ vs. $2.4 \pm 0.1 ; P=0.005)$. The tendency for 7 -NI to reduce GFR was significantly greater in rats receiving $\mathrm{BNZ}(P=$ 0.001 by 2 -way ANOVA). Coadministration of 7 -NI altered the response to BNZ, such that GFR was actually less in BNZ + 7-NI animals than in those treated with 7NI alone ( $P=0.001$ for the effect of $7-\mathrm{NI}$ on the response to post-BNZ treatment).

The role of macula densa NOS-I as a determinant of SNGFR was also tested by measuring SNGFR from the late proximal tubule during microperfusion with ATF at 38 and $8 \mathrm{~nL} / \mathrm{min}$, followed by ATF + SMTC at 38 and 8 $\mathrm{nL} / \mathrm{min}$, in 31 nephrons from 8 rats ( 4 control and 4 post-BNZ rats). Results during perfusion with ATF are described previously. Changing perfusate from ATF to ATF + SMTC significantly reduced SNGFR in both groups of animals at both perfusion rates. This impact of SMTC on SNGFR was significantly greater among rats treated with BNZ (SMTC reduced midpoint SNGFR by $19 \pm 3$ vs. $9 \pm 3 \mathrm{~nL} / \mathrm{min}$ in post-BNZ and control rats, 


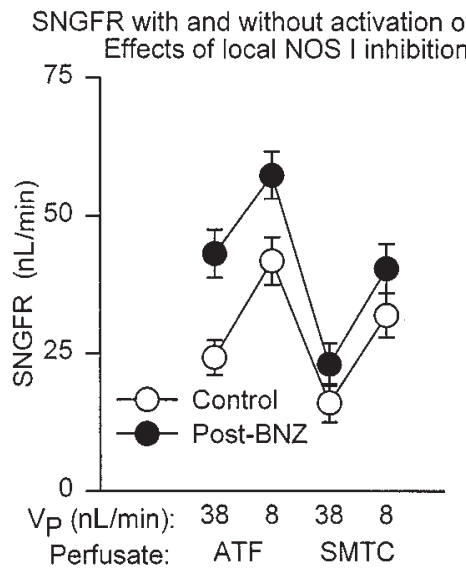

b

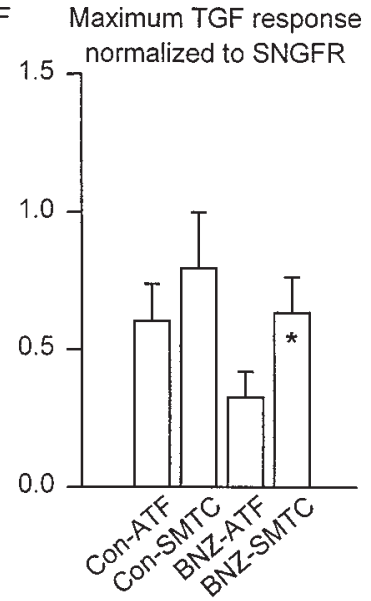

c

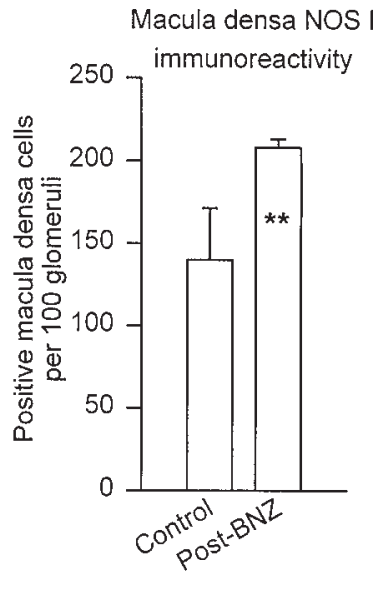

\section{Figure 3}

Macula densa NOS and TGF resetting. (a) Proximal SNGFR during microperfusion of the loop of Henle at $38 \mathrm{~nL} / \mathrm{min}$ (maximum TGF) and $8 \mathrm{~nL} / \mathrm{min}$ (minimum TGF, allowing for continued delivery of drug to the macula densa). Perfusion was with ATF or ATF + SMTC. The horizontal axis is arranged according to the chronology of the perfusion protocol. $V_{P=}$ microperfusion rate. Data shown are mean $\pm \mathrm{SEM}$ for control $(n=16)$ and $6-10$ hours post-BNZ nephrons $(n=15)$. When perfused with ATF, previous exposure to BNZ increased SNGFR, regardless of the state of TGF stimulation $(P=$ 0.002). SMTC reduced SNGFR at either perfusion rate in both groups ( $P=6 \times 10^{-8}$ for the effect of SMTC within subjects across both groups). The influence of SMTC was enhanced by BNZ, such that SMTC reduced the differences between BNZ and control $(P=0.02$ for the cross-term; group $\times$ SMTC with SNGFR averaged over both perfusion rates as dependent variable in ANOVA). (b) Increment in SNGFR that occurred when TGF was removed by reducing microperfusion from $38 \mathrm{~nL} / \mathrm{min}$ to $8 \mathrm{~nL} / \mathrm{min}$. Values were normalized to SNGFR averaged between the 2 measurements. ${ }^{*} P=$ 0.08 vs. BNZ-ATF. No other comparison approached significance. (c) Macula densa NOS-I immunoreactivity. ${ }^{*} P<0.05$ vs. control.

respectively; $P=0.02$ ). Within each group, the absolute magnitude of the effect of SMTC was roughly the same at each perfusion rate. However, in rats pretreated with BNZ, there was a tendency for SMTC to enhance the maximum TGF response, which was expressed as a fraction of the midpoint SNGFR (0.634 \pm 0.134 vs. $0.325 \pm$ 0.094; $P=0.08)$. No such tendency was noted in control animals $(0.796 \pm 0.204$ vs. $0.603 \pm 0.136 ; P=\mathrm{NS})$.

Treatment with BNZ caused a significant increase in the fraction of macula densa cells that stained positive with antibody to NOS-I (Figure 3), which correlated with the whole-kidney response to 7-NI and the nephron response to SMTC. Amounts of NOS-I mRNA, as determined by in situ hybridization, also suggested increased macula densa NOS in rats after BNZ, but the resolution using this technique was not as good as that received through immunohistochemistry, and the intergroup differences were not statistically significant.

To test for primary changes in loop of Henle reabsorption associated with BNZ, the relationship between early distal flow and VLP was studied by loop microperfusion with standard ATF or high-bicarbonate ATF in 47 nephrons from control and post-BNZ rats. Each nephron was perfused at 2 or 3 different rates ranging from 7 to $47 \mathrm{~nL} / \mathrm{min}$. Regression lines drawn from these data indicate that there was no effect of previous $\mathrm{BNZ}$ on loop reabsorption in nephrons perfused with standard ATF. Previous treatment with BNZ did, however, lead to increased reabsorption from nephrons perfused with high-bicarbonate ATF, indicating a slight increase in the capacity for bicarbonate-dependent transport in the descending limb of the loop of Henle in rats treated previously with BNZ (Figure 4).
Changes in ascending limb reabsorption will not affect the volume reabsorbed from the loop of Henle, except to the extent that effects on interstitial osmolarity affect water flux from the adjacent descending limb. To test for primary changes in ascending loop of Henle during $\mathrm{BNZ}$, the electrical conductivity of the early distal nephron was monitored during antegrade loop perfusion with standard ATF in 23 nephrons from control and post-BNZ rats. Measurements were made at 4 different perfusion rates in each nephron. Over the range of flows tested, early distal conductivity increased linearly with VLP. The relationship between VLP and early distal conductivity was not affected by previous treatment with $\mathrm{BNZ}(P=0.50$ for the effect of $\mathrm{BNZ}$ on the regression slope of conductivity vs. perfusion rate) (Figure 4).

To test for possible effects of BNZ on the reabsorptive machinery in the ascending limb of the loop of Henle, the $\mathrm{Na} / \mathrm{K} / 2 \mathrm{CL}$ transporter, BSC-1, was measured by immunoblotting in kidneys from 3 control and $3 \mathrm{BNZ}$ rats. The amount of BSC-1 protein detected by this method did not differ between the experimental groups (307 \pm 62 vs. $408 \pm 34$ densitometric units for BNZ and control rats, respectively). There was a tendency for animals treated with $\mathrm{BNZ}$ to express greater amounts of AQP1 (542 \pm 130 vs. $303 \pm 20$ densitometric units; $P=$ 0.20 by $t$ test $)$ and AQP2 (432 \pm 66 vs. $276 \pm 31$ densitometric units; $P=0.09$ by $t$ test), as determined by immunoblotting. However, these effects did not achieve statistical significance (Figure 5).

\section{Discussion}

The existence and functional significance of the TGF system have been established in publications from many lab- 
oratories. Most of this research has involved examining adjustments in glomerular filtration brought about within 2-3 minutes in order to compensate for abrupt changes in tubular flow. In such studies, the overall efficiency of the TGF system has been quantified, and the range of tubular flow rates over which TGF is maximally efficient has been shown to surround the natural tubular flow rate (1).

For the purposes of most micropuncture experiments, the behavior of TGF is considered to be static. However, it is obvious that TGF cannot be a static process. For example, the immediate action of TGF is to confer an inverse dependence of SNGFR on VLP. Therefore, any physiologic circumstance that permits or requires SNGFR and VLP to change in the same direction also requires a change in the behavior of TGF. Trivial examples of this include normal growth and development of the kidney, pregnancy, the renal response to changes in extracellular volume, changes in cardiovascular hemodynamics, and the single-nephron response to reduced renal mass. Furthermore, TGF adaptation to changing physiologic circumstances cannot be accounted for merely by increases or decreases in the maximum range of the TGF response or by changes in the TGF slope, because such changes would not account for the preservation of TGF efficiency that usually occurs (1).

Whereas it is clear that TGF must be capable of resetting, current understanding of how this occurs is minimal. In the past, we documented rightward TGF resetting within 1 hour when proximal flow was supplemented (2) or proximal reabsorption was inhibited (12). Therefore, it is possible to link TGF resetting to changes in the TGF signal that persist for 1 hour. The present studies were performed to examine the nature of TGF resetting over a period of 24 hours, a model arguably more analogous to certain real-life situations, such as the need for the kidney to grow or to respond to changes in salt intake. To induce TGF resetting, animals were repeatedly administered the proximal tubular diuretic BNZ. Rather than test for evi- dence of resetting under the continued influence of BNZ, we elected to look for evidence of resetting in the form of rebound hyperfiltration after the effects of $\mathrm{BNZ}$ on proximal reabsorption had subsided. The justification for this approach is as follows: Under the continuous influence of BNZ, SNGFR would likely remain somewhat less than baseline and VLP would likely remain somewhat greater than baseline, regardless of whether or not TGF has reset. When SNGFR and VLP change in opposite directions, single measurements of SNGFR and VLP obtained before and after these changes cannot reveal whether the nephron has shifted onto a new TGF curve or has moved along an original TGF curve. In contrast, SNGFR and VLP cannot change in the same direction unless there has been resetting of the TGF curve. Therefore, a simultaneous increase in both SNGFR and VLP to values above baseline would constitute positive proof of TGF resetting. We predicted that such proof might be obtained after the primary effect of BNZ had mostly subsided, but would not be revealed by data collected under the full ongoing influence of BNZ. Furthermore, the specificity of the results obtained with the chosen protocol would not be affected by tachyphylaxis to BNZ, inconstant tissue levels of BNZ, or reduced extracellular volume resulting from BNZ. In fact, the only assumptions required by the present approach regarding the pharmacokinetics or pharmacodynamics of $\mathrm{BNZ}$ are that $\mathrm{BNZ}$ did reduce proximal reabsorption and that it was less than maximally active at reducing proximal reabsorption during the time that physiologic data were being collected. A pictorial presentation of this argument is contained in Figure 6.

In this study, it was considered that TGF resetting in response to reduced proximal reabsorption might require increased NO formation by the macula densa. The basis for this consideration derives from a previous micropuncture study in which we demonstrated that the TGF curve shifted downward, leftward, and became more steep when macula densa NOS was blocked acutely by infusing
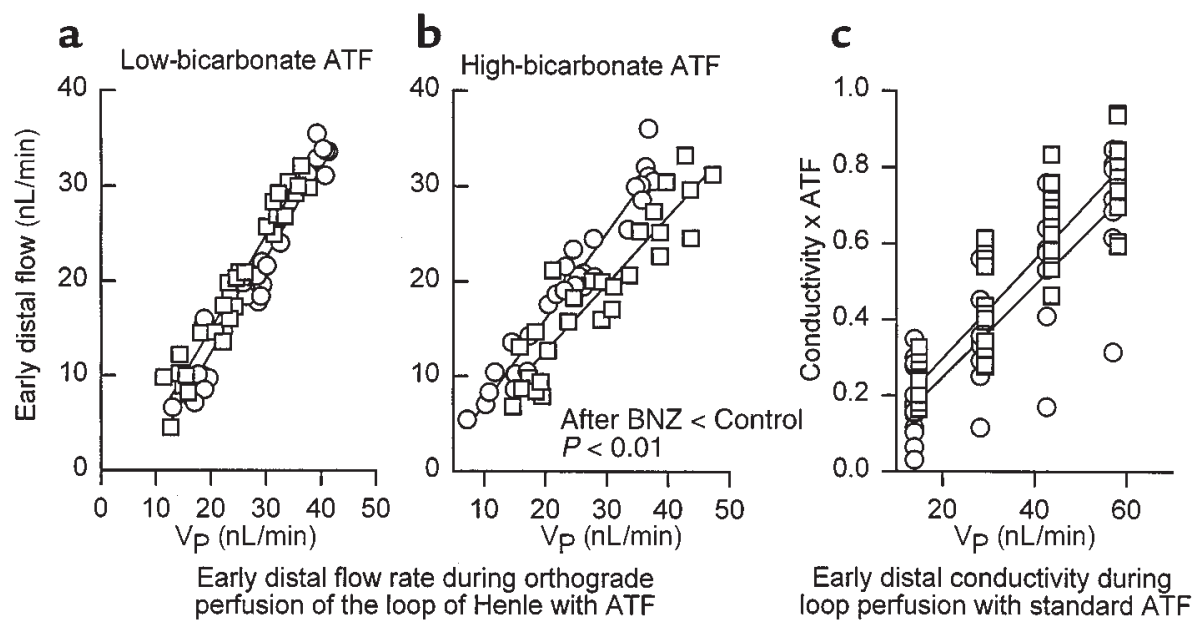

Figure 4

Loop of Henle reabsorption during loop perfusion. (a) Early distal flow rate during loop of Henle microperfusion with standard late ATF (10 mM HCO 3 ; low bicarbonate). (b) Early distal flow rate during perfusion with $25 \mathrm{mM} \mathrm{HCO}_{3}$ (high bicarbonate), demonstrating increased bicarbonate-dependent reabsorption in post-BNZ nephrons. (c) Electrical conductivity of the early distal nephron as a function of VLP in nephrons perfused with ATF. Conductivity is normalized to conductivity of standard late proximal ATF. The degree to which conductivity is reduced below ATF reflects the fraction of delivered solute reabsorbed from the water-impermeable ascending loop of Henle. Circles indicate controls; squares indicate post-BNZ nephrons. 


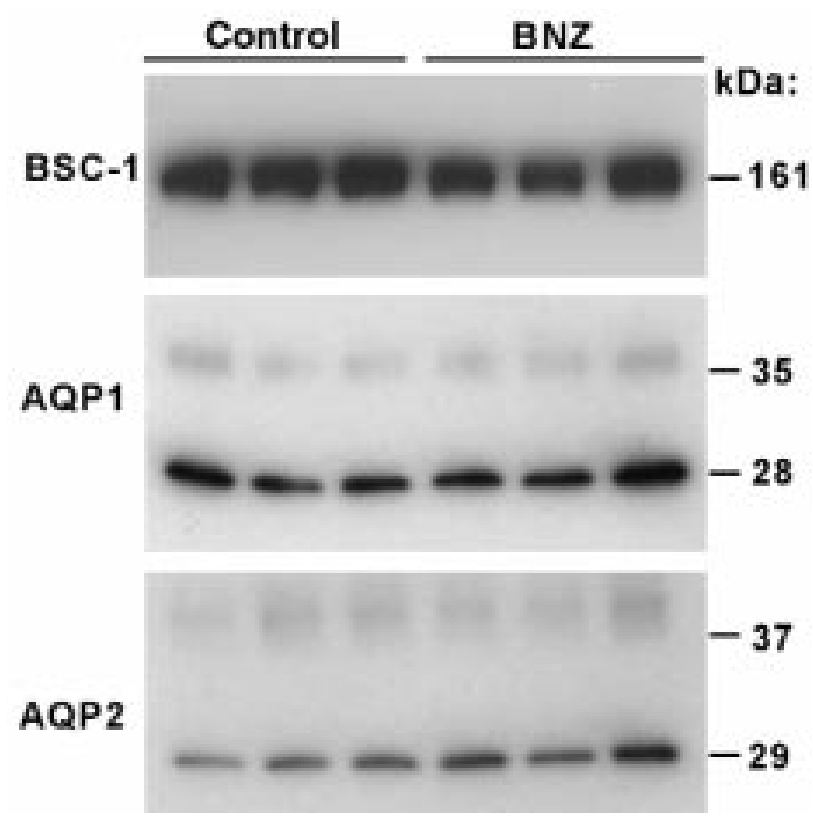

Figure 5

Immunoblot for BSC-1, APQ1, and AQP2 contents from kidneys of control or BNZ-treated rats.

the nonselective NOS blocker L-NMMA into the lumen of the free-flowing late proximal tubule (5). Those observations implied that NO formed by the macula densa of the euvolemic rat exerts a tonic upward pressure on SNGFR and a rightward pressure on the TGF curve, and suggested that a rightward and upward shift in the TGF curve would be the expected result if endogenous macula densa NO activity were to increase. Furthermore, others have suggested that the tonic influence of macula densa NOS over nephron function and TGF is enhanced when rats are fed a high-salt diet $(13,14)$, even though the high-salt diet reduces immunoreactive NOS in the macula densa $(15$, 16). There are few parallels to draw between rats receiving the diuretic $\mathrm{BNZ}$ and rats receiving a high-salt diet. However, these disparate treatments each cause flow to increase downstream from the early proximal tubule; each involves resetting of TGF to accommodate higher tubular flow; and each is associated with enhanced sensitivity of GFR to reduction by NOS inhibitors.

In the present study, NOS-I blockers were either administered systemically, along with each dose of BNZ, or added to the proximal tubular fluid of individual nephrons at the time of micropuncture. Both of these approaches yielded data consistent with idea that normal TGF resetting can be prevented by blocking macula densa NOS. As a caveat, NOS-I outside of the kidney performs a counterregulatory function in the control of renal sympathetic nerve traffic at the level of the central nervous system (17) and spinal cord (18). Because treatment with the diuretic could predispose to increased renal sympathetic nerve traffic, this role of NOS-I may have contributed to the subnormal GFR observed in rats treated with both 7$\mathrm{NI}$ and BNZ. However, the heightened sensitivity of the post-BNZ nephron to microperfusion with SMTC should be free of any such confounding influence.
Adding SMTC to ATF in control rats reduced SNGFR by similar amounts, regardless of whether TGF was being maximally or minimally activated at the time. The effects of SMTC on SNGFR are remarkably similar to effects that we reported previously for microperfusion with L-NMMA (5). When delivered into nephrons of post-BNZ rats, the effects of SMTC on SNGFR were similar in character, but of significantly greater magnitude than the effects of SMTC on control nephrons. As a result, the difference in SNGFR between control and BNZ groups was substantially reduced when each was perfused with SMTC (Figure 3). This confirms that macula densa NO exerts a greater tonic influence over SNGFR after BNZ, as would be expected if increased $\mathrm{NO}$ were responsible for resetting of TGF during BNZ treatment. To our knowledge, this experimental model is the first setting in which changes in macula densa NOS immunoreactivity and the magnitude of an associated physiologic response to NOS inhibition have not been paradoxical (13-16).

TGF is defined traditionally as a series of processes that occur in the JGA. However, when examined by micropuncture, it is most common to manipulate the TGF system from a site in the late proximal tubule. When this approach is taken, changes in loop of Henle function that alter the relationship between VLP and salt concentration at the macula densa may falsely suggest changes in the behavior of TGF. In this study, separate experiments were performed to test for the possibility that the apparent resetting of TGF could be due to a compensatory increase in loop of Henle transport. Indeed, previous treatment with BNZ was associated with increased loop of Henle reabsorption of both water and solute. However, the early distal flow rate remained elevated in the post-BNZ animal, and neither fractional reabsorption nor the ionic content of early distal tubular fluid was changed by previous treatment with BNZ. For a primary increase in loop reabsorption to account for the observed adaptation of TGF, the $\mathrm{NaCl}$ concentration at the macula densa during hyperfiltration must assume a value below baseline. If this criterion is not met, then increases in reabsorption noted during hyperfiltration are consistent with GTB or flow-dependent increases in transport, but not with a primary increase in transport sufficient to mimic the apparent resetting of TGF.

To normalize for differences in delivered load, changes in tubular function can be evaluated by examining fractional reabsorption. Free-flow micropuncture data alone do not yield a complete picture of the effects of previous BNZ administration on the loop of Henle. Therefore, we performed microperfusion studies that revealed that the volume of fluid reabsorbed from the loop of Henle was increased in post-BNZ animals, but only during perfusion with supraphysiologic concentrations of bicarbonate. This suggests an increased capacity for sodium-proton exchange in the nephron downstream from the perfusion site that is not brought to bear under natural conditions. One limitation of perfusing individual nephrons to study loop of Henle reabsorption is that the increment in volume reabsorbed per increment in applied VLP will underestimate the change that occurs when an equivalent increase in VLP affects a whole population of nephrons. This underestimation occurs because the hydro-osmotic 
force that governs water flux from the descending limb is influenced by the sum of the solute that is reabsorbed into the interstitium from the ascending limbs of many nearby nephrons, not just the index nephron. This accounts for the fact that the volume absorbed from the loop of Henle showed little dependence on VLP during microperfusion. However, using changes in early distal ionic content during loop perfusion as evidence for true changes in ascending limb function should not be encumbered by this problem, except when delivery to the ascending limb is affected. Given these caveats, the microperfusion data did not suggest a major primary increase in loop reabsorption after BNZ administration sufficient to account for an increase in SNGFR to the levels observed. This point was reinforced by the lack of any increase in the main thick ascending limb transporter, BSC-1. Therefore, whereas ambient reabsorption of both salt and water from the loop of Henle was increased after prolonged inhibition of proximal reabsorption, the events responsible for TGF resetting in this model must take place within the JGA rather than within the loop of Henle.

These studies demonstrate that during a sustained reduction in proximal reabsorption, events occur within the JGA that alter the behavior of TGF in such a way that both SNGFR and VLP become supranormal when proximal reabsorption returns toward normal. When TGF is depicted as a curve in the plane defined by VLP and SNGFR, this TGF adaptation is represented by a shift in the TGF curve that is upward and to the right (Figure 6). At the same time, there is only a small reduction in the maximum range of the TGF response, and the magnitude of the tonic influence exerted by TGF over ambient SNGFR is minimally affected. Thus, the steepest portion of the TGF relationship remains aligned with the actual tubular flow for optimal TGF efficiency. This is not to say that TGF efficiency is unaffected by previous treatment with BNZ. TGF efficiency, or the degree to which a TGF response succeeds in offsetting a particular disturbance in tubular flow, depends on the actual slope of the TGF curve, not merely on where the steep portion of the curve sits relative to the tubular flow (1). It is possible that the maximum slope of the TGF curve was affected by previous treatment with $\mathrm{BNZ}$, but the current studies were not designed to test this hypothesis.

The type of TGF resetting observed after BNZ treatment resembles the TGF adaptation that accompanies normal growth, in the sense that SNGFR and VLP both increase, whereas the macula densa signal, as indexed by the ionic content of the luminal fluid, remains normal. These events correlate with increased expression of NOSI activity in the macula densa and can be largely prevented by inhibition of NOS-I. Why is such adaptation important? The ability of TGF to reset is essential to maintaining the effectiveness of TGF as a mediator of short-term or dynamic autoregulation of nephron function. Also, and perhaps more importantly, the loss of normal TGF adaptation would curtail the ability to effect long-term control of the extracellular volume through changes in proximal tubular reabsorption. This is illustrated by the following example. When a step increase in salt intake is imposed on an animal that is salt-balanced, salt excretion will increase progressively until a new equilibrium is achieved a few days later. The amount of salt accrued in the course of reaching the new equilibrium will be proportional to the time required to

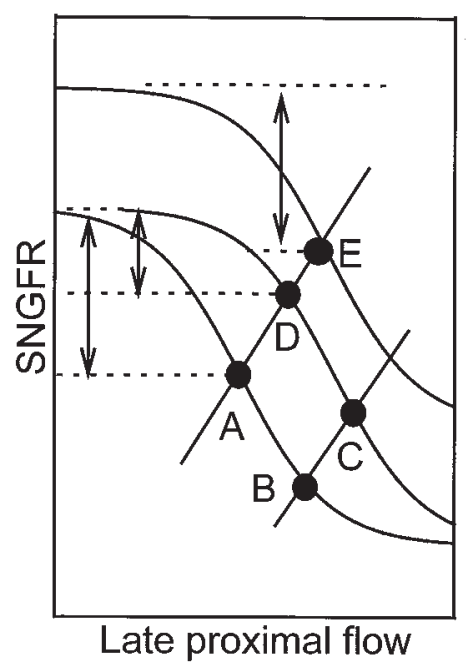

\section{Figure 6}

Graphic representation of the hypothesis to account for the observed effects of BNZ on ambient SNGFR and VLP, and to justify performing measurements after BNZ rather than during BNZ. The sigmoid curves refer to various TGF curves. Solid lines refer to the dependence of VLP on SNGFR owing to proximal GTB. Points A-E refer to the operating points where the various TGF and GTB curves intersect. Arrows refer to the PDD at each operating point. The control nephron operates at point $A$. The immediate effect of BNZ is to reduce proximal reabsorption, thereby moving the nephron to point $B$, where the TGF curve is relatively flat. Under the continuous influence of BNZ, the TGF curve resets rightward and the nephron moves to point $C$, where TGF efficiency is restored. If, as we hypothesize, it is the persistent activation of TGF that provides the stimulus for TGF resetting, then it follows that SNGFR $(C) \leq \operatorname{SNGFR}(A)$ and $\operatorname{VLP}(C)>\operatorname{VLP}(A)$. However, establishing these 2 inequalities cannot verify TGF resetting, because the same inequalities apply to the relationship between point $A$ and point $B$. Furthermore, administration of the diuretic BNZ might activate sodium-conserving mechanisms extrinsic to TGF, which would buffer the effect of BNZ on proximal reabsorption and exert a downward pressure on GFR through mechanisms that operate independently of TGF. Therefore, comparing point $A$ with point $C$ was not considered an appropriate means to test for TGF resetting during BNZ treatment. On the other hand, allowing proximal tubular reabsorption to increase by withdrawing BNZ would move the operating point to point $D$, where SNGFR $(D)>\operatorname{SNGFR}(A)$ and $\operatorname{VLP}(D)>\operatorname{VLP}(A)$. These 2 inequalities cannot both be satisfied unless there is rightward and/or upward resetting of TGF. Furthermore, increases in SNGFR are contrary to any expected outcome mediated through the effects of BNZ on extracellular volume. Therefore, establishing that SNGFR (D) > SNGFR (A) and $\operatorname{VLP}(\mathrm{D})>\operatorname{VLP}(\mathrm{A})$ is sufficient to validate that TGF has reset. However, it cannot be determined from the coordinates of the operating point whether the resetting is primarily rightward (point D) or rightward and upward (point E). This latter issue can be addressed by the testing for changes in the PDD and by measuring SNGFR from the proximal tubule during minimum and maximum stimulation of TGF. IfTGF resets purely rightward during $B N Z$, then the increase in ambient SNGFR after BNZ is withdrawn will be associated with a subnormal PDD (point D). If the PDD is not subnormal after withdrawal of BNZ, then there must be an upward component to the resetting of TGF (point E). In the present experiments, the PDD was unaffected by previous treatment with BNZ, inferring that the TGF curve had shifted both upward and rightward. 
reach that equilibrium (19). The natriuretic response to salt loading normally includes a shift in proximal GTB, i.e., reduced proximal reabsorption at any given SNGFR (20). This shift occurs because the proximal tubule is sensitive to neurohumoral signals that are linked to changes in total body salt. However, the natriuretic effect of any given shift in proximal GTB depends on whether there is simultaneous resetting of TGF. Whereas the nature of TGF is to buffer any short-term disturbance in GTB, thereby stabilizing VLP, a rightward resetting of TGF will reduce the increment in total body salt required to bring about a given increment in VLP through reduced proximal reabsorption. Thus, rightward resetting of TGF will shorten the time required to reach equilibrium after an increment in salt intake, and will lessen the long-term impact of salt intake on steady-state salt content. Conversely, impaired TGF resetting would prolong the time required to achieve equilibrium salt balance in response to increased salt intake, thus increasing the effect of salt intake on steady-state total body salt content. Thus, the phenomenon of TGF resetting has obvious consequences for the long-term control of blood pressure.

Most research concerning TGF in pathophysiologic states has focused on assessing the acute TGF response. Such studies have demonstrated that TGF efficiency or the maximum TGF response can be suppressed or enhanced in certain conditions. This study confirms the previous observation that TGF normally resets rightward during a sustained reduction in proximal reabsorption (12), and demonstrates that this resetting can persist for many hours after the stimulus for resetting is removed. Furthermore, this form of TGF resetting appears to require generation of $\mathrm{NO}$ in the macula densa. Finally, these results suggest that time be viewed as a relevant dimension when considering the potential pathophysiologic consequences of altered interactions between the tubule and glomerulus.

\section{Acknowledgments}

This work was supported by grant DK-28602 from the National Institutes of Health, and by the US Depart- ment of Veterans Affairs.

1. Thomson, S.C., and Blantz, R.C. 1993. Homeostatic efficiency of tubuloglomerular feedback in hydropenia, euvolemia, and acute volume expansion. Am. J. Physiol. 264:F930-F936.

2. Thomson, S.C., Blantz, R.C., and Vallon, V. 1996. Increased tubular flow induces resetting of tubuloglomerular feedback in euvolemic rats. Am. J. Physiol. 270:F461-F468.

3. Tucker, B.J., Steiner, R.W., Gushwa, L.C., and Blantz, R.C., 1978. Studies on the tubulo-glomerular feedback system in the rat. The mechanism of reduction in filtration rate with benzolamide. J. Clin. Invest. 62:993-1004.

4. Blantz, R.C., and Tucker, B.J. 1978. Measurements of glomerular dynamics. In Methods in pharmacology. M. Martinez-Maldonado, editor. Plenum Press. New York, NY. 141-163.

5. Vallon, V., and Thomson, S.C. 1995. Inhibition of local nitric oxide synthase increases the homeostatic efficiency of tubuloglomerular feedback. Am. J. Physiol. 38:F892-F899.

6. Bredt, D.S., et al. 1991. Cloned and expressed nitric oxide synthase structurally resembles cytochrome P-450 reductase. Nature. 351:714-718.

7. Bachmann, S., Bosse, H.M., and Mundel, P. 1995. Topography of nitric oxide synthesis by localizing constitutive NO synthases in mammalian kidney. Am. J. Physiol. 268:F885-F898.

8. Bachmann, S., Metzger, R., and Bunnemann, B. 1990. Tamm-Horsfall protein-mRNA synthesis is localized to the thick ascending limb of Henle's loop in rat kidney. Histochemistry. 94:517-523.

9. Lowry, O.H., Rosebrough, N.J., Farr, A.L., and Randall, R.J. 1951. Protein measurement with the folin phenol reagent. J. Biol. Chem. 193:265-275.

10. Ecelbarger, C.A., et al. 1997. Role of renal aquaporins in escape from vasopressin-induced antidiuresis in rat. J. Clin. Invest. 99:1852-1863.

11. Ecelbarger, C.A., et al. 1996. Localization and regulation of the rat renal $\mathrm{Na}(+)-\mathrm{K}(+)-2 \mathrm{Cl}-$ cotransporter, BSC-1. Am. J. Physiol. 271:F619-F628.

12. Thomson, S.C., Vallon, V., and Blantz, R.C. 1997. Acute reduction in proximal reabsorption induces resetting of tubuloglomerular feedback. Am.J. Physiol. 273:R1414-R1420.

13. Welch, W.J., and Wilcox, C.S. 1997. Role of nitric oxide in tubuloglomerular feedback: effects of dietary salt. Clin. Exp. Pharmacol. Physiol. 24:582-586.

14. Wilcox, C.S., and Welch, W.J. 1996. TGF and nitric oxide: effects of salt intake and salt-sensitive hypertension. Kidney Int. Suppl. 55:S9-S13.

15. Tojo, A., Madsen, K.M., and Wilcox, C.S. 1995. Expression of immunoreactive nitric oxide synthase isoforms in rat kidney. Effects of dietary salt and losartan. Jpn. Heart J. 36:389-398.

16. Bosse, H.M., Bohm, R., Resch, S., and Bachmann, S. 1995. Parallel regulation of constitutive NO synthase and renin at JGA of rat kidney under various stimuli. Am. J. Physiol. 269:F793-F805.

17. Iida, N. 1999. Nitric oxide mediates sympathetic vasoconstriction at supraspinal, spinal, and synaptic levels. Am. J. Physiol. 276:H918-H925.

18. Zhang, K., Mayhan, W.G., and Patel, K.P. 1997. Nitric oxide within the paraventricular nucleus mediates changes in renal sympathetic nerve activity. Am. J. Physiol. 273:R864-R872.

19. Walser, M. 1985. Phenomenological analysis of electrolyte and water homeostasis. In The kidney: physiology and pathophysiology. D.W. Seldin and G. Giebisch, editors. Raven Press. New York, NY. 3-15.

20. Daugherty, T.M., Ueiki, I.F., Nicholas, D.P., and Brenner, B.M. 1973. Renal response to chronic intravenous salt loading in the rat. J. Clin. Invest. 52:21-31. 